\title{
Asymptotic Relationships between Systems of Volterra Equations.
}

\author{
JoHN A. Nohel (*)
}

\begin{abstract}
Summary. - Stability type results concerning continuous solutions of perturbed systems of Volterra equations as well as theorems concerning the asymptotic equivalence of such perturbed and unperturbed systems are established.
\end{abstract}

1. Introduction. - Consider the perturbed system of Volterra equations

$$
x(t)=f(t)-\int_{0}^{t} a(t, s)[x(s)+g(s, x(s))] d s \quad(t \geq 0)
$$

where $x, f, g$ are $n$-component vector functions, $a$ is a $n$ by $n$ matrix, and the perturbation $g$ is regarded as small in various senses; in all cases $g(t, x)=\circ(|x|)$ uniformly in $t$ as $|x| \rightarrow 0$ will be sufficient, except in Theorem 4. Our purpose is to compare in various ways solutions of (1.1) with those of the unperturbed linear system

$$
y(t)=f(t)-\int_{0}^{t} a(t, s) y(s) d s
$$

In doing this we generalize stability type results for continuous solutions in [1], [2], where a stronger hypothesis than the one used here is required of $g$. However, here we give up uniqueness. This is done in Theorems 1 and 2. Our main result, Theorem 3, provides a criterion for the asymptotic equivalence of (1.1), (12). The first part of Theorem 3 partially extends a result of A. STRaUss [3; Theorem 2$]$, where a considerably stronger hypothesis is required of the kernel $a(t, s)$, than is needed here; on the other hand we require an additional hypothesis of the nonlinear terms $g$ in the general case.

(*) Entrata in Redazione il 12 gennaio 1971

Supported by AROD-Durham. 
The second part is apparently new. In the important special case conside. red in Theorem 4:

$$
|g(t, x)| \leq \lambda(t)|x|^{\sigma} \quad(0 \leq \sigma<1)
$$

where $\lambda(t)$ is a bounded continuous function on $0 \leq t<\infty$ tending to zero as $t \rightarrow \infty$, the additional hypothesis needed to prove the first part of Theorem 3 is dropped and the proof is carried out by an elementary argument based on obtaining an a priori estimate for solutions of (1.1). Theorem 3 and 4 may be regarded as a generalization to VoLterra equations of classical results on asymptotic equivalence due primarily to $\mathrm{N}$. LEVINSON [4] and H. WEYL [5] (see especially F. Brauer and J.S.W. Wong [6] for a recent and general form of these results) in ordinary differential equations. The author is grateful to F. Braugr for pointing out the most recent version of these results to him, and to R.K. Mile er and P. Rabinowitz for useful discussions concerning Theorems 3 and 4 .

The main tool in proving our results is the following form of the SCHAUdER-TrohoNoff fixed point theorem. Its elementary proof may be found in COPPEL [7].

Theorem. - Let $C(J)$ denote the set of functions which are continuous on the interval $J$ (bounded or unbounded). Let $F$ denote the subset of these functions $x(t)$ such that $|x(t)| \leq \mu(t)$ for all $t \in J$, where $\mu$ is a fixed positive continuous function on $J$. Let $T$ be a mapping of $F$ into itself such that

(i) $T$ is continuous (in the sense that if $x_{n} \in F, n=1,2, \ldots, x_{n} \rightarrow x$ uniformly on compact subsets of $J$, then $T x_{n} \rightarrow T x$ uniformly on compact subsets of $J)$

(ii) the functions in the image set TF are equicontinuous and bounded at each point of $J$.

Then the mapping $T$ has at least one fixed point in $F$. (It is important to notice that the functions in $T F$ need not be aniformly equicontinuous on $J$ ).

2. Summary of Results. - We first require, as in [1] and [3] several con-) cepts and formulae which will be used throughout The resolvent kernel $r(t, s$ corresponding to the kernel $a(t, s)$ in (1.1) is defined as the (unique) solution of the resolvent system

$$
r(t, s)=a(t, s)-\int_{s}^{t} a(t, u) r(u, s) d u
$$


this solution exists (and is locally integrable in $(t, s)$ ) if the kernel $a(t, s)$ is locally integrable in $(t, s)$ for $0 \leq s \leq t<\infty$. The solution of the linear sy. stem (1.2) is expressible as

$$
y(t)=f(t)-\int_{0}^{t} r(t, s) f(s) d s
$$

under various hypotheses concerning the given function $f$. Moreover, it can be shown that the system (1.1) is equivalent to the integral equation

$$
x(t)=y(t)-\int_{0}^{t} r(t, s) g(s, x(s)) d s,
$$

where $y(t)$ is given by (2.2). We remark that the proof of the equivalence is a rather long but elementary exercise involving the use of (2.1). In going from (1.1) to (2.3) ase is made of the fact that if $a, \mathrm{r}$ satisfy (2.1) then

$$
\int_{s}^{t} a(t, u) r(u, s) d u=\int_{s}^{t} r(t, u) a(u, s) d u .
$$

The motivation for introdueing the resolvent kernel, and the equivalent system (2.3) as well as various hypotheses concerning $r(t, s)$, notably (2.4) be. low, which follow as well as several applications and examples may be found in [1] and [3].

Unless stated explicity otherwise we assume the following:

(H) $r(t, s)$ is locally integrable in $(t, s)$ for $0 \leq s \leq t<\infty$.

$\left(H_{2}\right) \quad g(t, x)$ is continuous in $(t, x)$ for $0 \leq t<\infty,|x|<\infty$ and $g(t, 0)=0$.

$\left(H_{3}\right)$ for every $\varepsilon>0$ there exists a $\delta>0$ such that $|g(t, x)| \leq \varepsilon|x|$ uniformly in $t$, whenever $|x| \leq \delta$. Here || is any convenient norm in Euclidean $n$-space.

Let $B C$ denote the class of bounded and continuous functions on $0 \leq t<\infty$. If $\varphi \in B C$ define

$$
\|\varphi\|=\sup _{0 \leq t<\infty}|\varphi(t)|
$$


THEOREM 1. - Suppose $y(t) \in B C$ is a solution of the linear system in (1.2). Let there exist a constant $B>0$ such that

$$
\int_{0}^{t}|r(t, s)| d s \leq B(t \geq 0)
$$

and let

$$
\lim _{h \rightarrow \infty}\left[\int_{i}^{t+h}|r(t+h, s)| d s+\int_{0}^{t} \mid r(t+h, s)-r(t, s)_{i}^{\mid} d s\right]=0
$$

for each $t>0$. Then for every $\lambda, 0<\lambda<1$, there exists a $\varepsilon_{0}>0$ such that $0<\varepsilon \leq \varepsilon_{0}$ and $\|y\| \leq \lambda \varepsilon$ together imply that there exists at least one solution $x(t)$ of the perturbed system (1.1) such that $x(t) \in B C$ and $\|x\| \leq \varepsilon$.

The condition $\|y\| \leq \lambda \varepsilon$ is natarally satisfied by requiring $f \in B C$ and $\|f\| \leq \lambda \varepsilon$ $(1+B)^{-1}$, as may be seen from (2.2). Thus Theorem 1 may be interpreted as a stability result in which $f$ plays the role of initial conditions.

If $g$ happens to satisfy a LIPSoHITz condition (with a constant $M$ that is arbitrary as opposed to the situation in [1]) the solution $x(t)$ of Theorem 1 is unique.

As an immediate consequence of Theorem 1 we have the following result which may be regarded as an asymptotic stability theorem.

CoRoLlany 1.1. - If in addition to the hypotheses of Theorem $1 \lim _{t \rightarrow \infty} y(t)=0$ and for each $T>0$

$$
\lim _{t \rightarrow \infty} \int_{0}^{T}|r(t, s)| d s=0
$$

then the solution $x(t)$ satisfies $\lim _{i \rightarrow \infty} x(t)=0$.

Note that $\lim _{t \rightarrow \infty} f(t)=0,(2.2)$, and (2.6) imply that $\lim _{t \rightarrow \infty} y(t)=0$.

Theorem 1 and Corollary 1.1 extend Theorem 3 and Corollary 3.1 of [1] at the expense of giving up uniqueness. Several applications discussed in [1], in particular the example (Theorem 6) hold in the present, more general, setting. Theorem 1 can also be proved by applying a general result recently established independently by Miller [8].

We next extend by a similar technique the result established in [2].

This concerns the VOLTERRA-FredHoLm system

$$
\left.x(t)=f(t)+\int_{0^{-}}^{t} v(t, s) g(s, x(s)) d s+\int_{0}^{\infty} k(t, s) h \cdot s, x(s)\right) d s
$$


for $t \geq 0$. Note that this is not a perturbation of a linear system; (2.7) arises naturally in studying boundary value problems on the infinite half line. The functions $f, g, h$ are given $n$-vectors, while $v$ and $k$ are given $n$ by $n$ ma. trices. We shall not introduce the resolvent kernel; instead we shall make a fairly strong hypothesis concerning the kernel $v$ and $k$ as we did in [2].

Theorem 2. - Let $f \in B C$ and let the kernel $v, k$ satisfy $H_{1}$. Let $v$ satisfy (2.4), (2.5) and let there exist a constant $K>0$ such that

$$
\int_{0}^{\infty}|k(t, s)| d s \leq K<\infty(0 \leq t<\infty)
$$

further, let

$$
\lim _{h \rightarrow 0} \int_{0}^{\infty}|k(t+h, s)-k(t, s)| d s=0
$$

for every $t>0$. Let $g, h$ satisfy $H_{3}$. There exists a number $\varepsilon_{0}>0$ such that to every $0<\varepsilon \leq \varepsilon_{0}$ there corresponds a $\delta>0$ with the property that $\|f\| \leq \delta$ implies the existence of a sotution $x(t) \in B C$ of (2.7) such that $\|x\| \leq \varepsilon$. If in addition $\lim _{t \rightarrow \infty} f(t)=0$ and if $v$ also satisfies (2.6) and if $k$ also satisfies

$$
\lim _{t \rightarrow \infty} \int_{0}^{\infty}|k(t, s)| d s=0
$$

then the solution $x(t)$ satisfies $\lim _{t \rightarrow \infty} x(t)=0$.

As in Theorem 1 , if both $g$ and $h$ satisfy a LipsoHitz condition the solution $x$ in Theorem 2 is unique. Notice that Theorem 2 holds if either $v$ or $k$ in (2.7) are identically zero. However, Theorem 2 is a strictly nonlinear result. There are various ways to see that Theorem 2 does not hold in general for linear systems. Take for instance $k(t, s) \equiv 0, f(t) \equiv f_{0}$ (a constant), $g(t, x)=\rho x$ where $\rho$ is a fixed constant, and

$$
v(t, s)=\exp [-2(t-s)]-\exp [-(t-s)]
$$

Then $v$ and $k$ satisfy the hypotheses of Theorem 2 , but $g$ does not. The unique solution of (2.7) in this case is

$$
x(t)=c_{1} \exp \left(\rho_{1} t\right)+c_{2} \exp \left(p_{2} t\right)+\frac{2}{\rho+2} f_{0}(p \neq-2)
$$

where $c_{1}, c_{2}$ are fixed constants (depending on $\left.\rho_{1}, \rho_{2}, f_{0}\right)$. If $\rho<-2$ one has $p_{1}>0, p_{2}<0, c_{1} \neq 0$ and the solution $x(t)$ is not bounded on $0 \leq t<\infty$. 
(If $\rho=-2$, the corresponding solution is also unbonnded). Examples similar to this one, but for integrodifferential equations were constructed by LeviN $[9 ;$ p. 372$]$.

Theorem 2 not only extends the result of [2], but the present proof also corrects an error in the proof in [2], where it was asserted that the integral operator $B$ defined by the kernel $k(t, s)$ is completely continuous on the space $S(\varepsilon)$ of functions $\varphi \in B C$ and $\|\varphi\| \leq \varepsilon$. This, however, is not the case and the. refore the Lemma of KRAsNoseLsKII used in [2] is not applicable (moreover, it was not needed since an independent proof of the result stated in [2] is easily constructed).

Our main resuit generalizes Theorem 1 and Corollary 1.1 above, as well as Theorem 2 of [3] in several ways. In place of $H_{2}, H_{3}$ we now suppose

$H_{4} \quad\left\{\begin{array}{c}g(t, x) \text { is continuous in }(t, x) \text { for } 0 \leq t<\infty,|x|<\infty \text { and } \\ |g(t, x)| \leq\left\{\begin{array}{cc}\lambda(t)|x| \text { if }|x| \geq 1 \\ \lambda(t) & \text { if }|x|<1\end{array}\right.\end{array}\right.$

where $\lambda(t) \geq 0$ is a fixed bounded continuous function on $[0, \infty)$ such that $\lim _{t \rightarrow \infty} \lambda(t)=0$ and such that $\|\lambda\| B \leq \frac{1}{2}$ where $B>0$ is the a priori constant defined in (2.4).

Theоnem 3. - Let $g$ satisfy $H_{4}$; let the resolvent keruel $r(t, s)$ satisfy $H_{1},(2.4),(2.5),(2.6)$. Let $y(t) \in B C$ be a fixed solution of the unperturbed system (1.2). Then there exists a solution $x(t) \in B C$ of the perturbed system (1.1) such that

$$
\lim _{t \rightarrow \infty}(x(t)-y(t))=0
$$

Conversely, given a solution $x(t) \in B C$ of (1.1) there exists a solution $y(t) \in B C$ (1.2) such that (2.11) holds; i.e. the systems (1.1) and (1.2) are asymptotically equivalent.

The remarks in [3] concerning the implications of Theorem 3 remain, of course, valid. In particular $H_{4}$ includes as a special case the condition

$$
|g(t, x)| \leq \lambda(t)|x|^{\alpha}(0 \leq \alpha \leq 1)
$$

where $\lambda(t)$ is as in $H_{4}$. However, the condition $\lim \lambda(t)=0$ cannot be improved to $\int_{0}^{\infty} \lambda(t) d t<\infty$, as shown by Example $3 \stackrel{t \rightarrow \infty}{\text { of }}[3]$. 
The first part of Theorem 3 partially generalizes Theorem 2 of [3] because in [3] the original kernel $a(t, s)$ must satisfy a much more stringent hypothesis (see $H_{1}$ in [3]) than is needed here. The most serious of these is

$$
\sup _{0 \leq \leq \leq T} \int_{0}^{t}|a(t, s)| d s<\infty
$$

for every $T>0$. This hypothesis need not be satisfied for the prototype example $a(t, s)=A(t-s) B(s)$ discussed in [1, p. 68\%], where $A(t)$ is completely monotonic on $0<t<\infty$ and $B(t) \geq 0$ is of class $B C$, and to which our results are applicable.

Finally, we return to the special case

$$
|g(t, x)| \leq \lambda(t)|x|^{\sigma} \quad(0 \leq \sigma<1)
$$

where $\lambda(t) \geq 0$ is as in $\left(H_{4}\right)$, but the condition $\|\lambda\| B \leq \frac{1}{2}$ is dropped.

TheоRem 4. - Let $g$ satisfy (2.12) and let the remaining assumptions of Theorem 3 be satisfied. Then the systems (1.1) and (1.2) are asymptotically equivalent in the sense of Theorem 3.

3. Proof of Theorem 1 and Corollary 1.1. - Fix a number $\beta>0$ such that $\beta B<1-\lambda$. By $H_{3}$ choose the number $\varepsilon_{0}>0$ in the statement of Theo. rem 1 such that

$$
|g(t, x)| \leq \beta|x| \quad\left(|x| \leq \varepsilon_{0}\right)
$$

uniformly in $t$. Let $0<\varepsilon \leq \varepsilon_{0}$ and consider the set

$$
S_{\varepsilon}=\{\varphi \in B C:\|\varphi\| \leq \varepsilon\} .
$$

Define the operator $R$ by the relation

$$
(R \varphi)(t)=y^{\prime}(t)-\int_{0}^{t} r(t, s) g(s, \varphi(s)) d s
$$

for any function $\varphi \in B C$. We assert:

(i) $R$ maps $S_{\varepsilon}$ into itself for $0 \leq \varepsilon \leq \varepsilon_{0}$. 
For, nsing (2.4), (3.1)

$$
\begin{aligned}
|R \varphi(t)| & \leq|y(t)|+\int_{0}^{t}|r(t, s)| \beta|\varphi(s)| d s \\
& \leq\|y\|+\beta \sup _{0 \leq s \leq t}|\varphi(t)| B \leq \lambda \varepsilon+\beta \varepsilon B .
\end{aligned}
$$

Thus

$$
\|R \varphi\| \leq \lambda \varepsilon+(1-\lambda) \varepsilon=\varepsilon \quad\left(0 \leq \varepsilon \leq \varepsilon_{0}\right)
$$

and $R S_{\varepsilon} \subseteq S_{\varepsilon}$

(ii) The mappinq $R$ is continuous on $S_{\varepsilon}$.

For, if $\varphi_{n} \in S_{\varepsilon}$ and $\left\{\varphi_{n}\right\} \rightarrow \varphi$ uniformly on every compact subset of $[0, \infty)$, then from (3.2), (2.4)

$$
\begin{aligned}
\left|(R \varphi)(t)-\left(R \varphi_{n}\right)(t)\right| & \leq \int_{0}^{t}|r(t, s)|\left|g(s, \varphi(s))-g\left(s, \varphi_{n}(s)\right)\right| d s \\
& \leq \sup _{\substack{0 \leq s i}}\left|g(s, \varphi(s))-g\left(s, \varphi_{n}(s)\right)\right| B
\end{aligned}
$$

which tends to zero as $n \rightarrow \infty$, uniformly on compact subsets of $[0, \infty)$ by $\left(H_{2}\right)$.

(iii) The functions in the inage set $R S_{\varepsilon}, 0 \leq \varepsilon \leq \varepsilon_{0}$, are equicontinuous and bounded at every point of $[0, \infty)$.

For, the boundedness is already proved in (i). For the pointwise equicontinuity let $t_{0} \in\left[0, \infty\right.$ ) and let $t>t_{0}$ (the same argument applies to $t<t_{0}$ ). Then from (3.2) one has

$$
\begin{gathered}
(R p)(t)-\left(R_{\varphi}\right)\left(t_{0}\right)=y\left(t_{0}\right)+\int_{0}^{t_{0}} r\left(t_{0}, s\right) g(s, \varphi(s)) d s \\
-\int_{0}^{t} r(t, s) g(s, \varphi(s)) d s \\
=y(t)-y\left(t_{0}\right)+\int_{0}^{t}\left[r\left(t_{0}, s\right)-r(t, s)\right] g(s, \varphi(s)) d s \\
-\int_{t_{0}}^{t} r(t, s) g(s, \varphi(s)) d s .
\end{gathered}
$$


Thus

$$
\begin{aligned}
& \mid(R \varphi)(t)-\left(R \varphi\left(t_{0}\right)|\leq| y(t)-y\left(t_{0}\right) \mid\right. \\
& \left.\quad+\sup _{0 \leq s \leq t}|g(s, \varphi(s))|\left|\int_{0}^{t_{0}}\right| r\left(t_{0}, s\right)-r(t, s)\left|d s+\int_{s_{0}}^{t}\right| r(\mathbf{t}, s) \mid d s\right],
\end{aligned}
$$

and by $H_{3},(3.1)$ one easily has

$$
\begin{aligned}
\mid(R \varphi)(t)- & (R \varphi)\left(t_{0}\right) \mid \leq \\
& \left|y(t)-y\left(t_{0}\right)\right|+\beta \varepsilon_{0}\left[\int_{0}^{t_{0}}\left|r\left(t_{0}, s\right)-r(t, s) d s+\int_{t_{0}}^{t}\right| r(t, s) \mid d s\right] .
\end{aligned}
$$

Let $\eta>0$ be given. Choose $\delta_{1}>0$ so that

$$
\left|y(t)-y\left(t_{0}\right)\right| \leq \frac{\eta}{2} \text { for }\left|t-t_{0}\right| \leq \delta_{1} .
$$

By (2.5) choose $\delta_{2}>0$ so that

$$
\begin{array}{r}
\int_{0}^{t_{0}}\left|\boldsymbol{r}(t, s)-\boldsymbol{r}\left(t_{0}, s\right)\right| d s+\int_{t_{0}}^{t}|r(t, s)| d s \leq \frac{\eta}{2\left(1+\beta \varepsilon_{0}\right)} \\
\text { for }\left|t-t_{0}\right| \leq \delta_{2} .
\end{array}
$$

Let $\delta=\min \left(\delta_{1}, \delta_{2}\left(\right.\right.$ (since $\delta_{1}, \delta_{2}$ may depend on $t$ so does $\left.\delta\right)$. Then

$$
\mid\left(R \varphi(t)-R \varphi\left(t_{0}\right) \mid \leq \eta \quad \text { whenever }\left|t-t_{0}\right| \leq \delta\right.
$$

establishing the pointwise equicontinuity of the functions in $R T_{\varepsilon}$.

Thas by the Soradder-Tyohonoff fixed point theorem there exists a function $\tilde{\varphi} \in S_{\varepsilon}$ such that $R \tilde{\varphi}=\tilde{\varphi}$ or such that

$$
\tilde{\varphi}(t)=y(t)-\int_{0}^{t}(r t, s(g(s, \tilde{\varphi}(s)) d s \quad(0 \leq t<\infty)
$$

and $\|\tilde{\varphi}\| \leq \varepsilon$. Since (2.3) and (1.1) are equivalent systems, this completes the proof of Theorem 1 .

To prove Corollary 1.1 we may define

$$
S_{0}=\left\{\varphi: \varphi \in S_{\mathrm{s}} \text { and } \lim _{t \rightarrow \infty} \varphi(t)=0\right\}
$$


Evidently $S_{0}$ is a closed subset of $S_{8}$ under the uniform norm.

Thus it suffices to show that $R S_{0} \subseteq S_{0}$, i.e. $\lim _{t \rightarrow \infty}(R \rho)(t)=0$ whenever $\lim _{t \rightarrow \infty} \varphi(t)=0$.

For a proof of this elementary fact see [1, p. 685]. Alternatively, suppose $\lim _{t \rightarrow \infty} x(t) \neq 0$, where $x(t)$ is a solution of $(2.3)$ in $S_{\varepsilon}$, and let

$$
\mu=\lim _{t \rightarrow \infty} \sup |x(t)|>0
$$

Fix a number $\gamma$, where $1-\lambda<\gamma<1$ and where $\lambda$ is the number in Theorem 1. Choose $T>0$ so large that $\left|\boldsymbol{x}^{\prime}(t)\right| \leq \mu^{/ \gamma}$ whenever $t \geq T$. Then (2.3), (3.1) imply that

$$
\begin{aligned}
|x(t)| & \leq|y(t)|+\int_{0}^{T}|r(t, s)||g(s, x(s))| d s+\int_{T}^{t}|r(t, s)||g(s, x(s))| d s \\
& \leq|y(t)|+\beta \varepsilon \int_{0}^{T}|r(t, s)| d s+\beta \frac{\mu}{\gamma} \int_{T}^{t}|r(t, s)| d s .
\end{aligned}
$$

Taking the lim sup one obtains from the hypothesis and (2.4), (2.6)

$$
\mu \leq 0+0+\beta \frac{\mu}{\gamma} B<\frac{\mu}{\gamma}(1-\lambda)<\mu
$$

which is a contradiction and the proof of Corollary 1.1 is complete.

4. Proof of Theorem 2. - For $\varphi \in S_{\varepsilon}$ define the operator $T$ by the relation

$$
(T \varphi)(t)=f(t)+\int_{0}^{t} v(t, s) g(s, \varphi(s)) d s+\int_{0}^{\infty} h(t, s) h(s, \varphi(s)) d s .
$$

We again show that $T$ satisfies the hypothesis of the SOHAUDER-TrchonofF fixed point theorem. We assert

(i) $T S_{\varepsilon} \subseteq S_{\varepsilon}$ for $0 \leq \varepsilon \leq \varepsilon_{0}$.

Fix $\zeta>0$ such that $K \zeta<1$. By $H_{3}$ pick a number $\sigma_{1}>0$ such that $|\boldsymbol{h}(t, x)| \leq \zeta|x|$ for $|x| \leq \sigma_{1}$, uniformly in $t$. Let $\gamma=\frac{1-K \zeta}{2 B}$ and choose a number $\sigma_{2}>0$ such that $|g(t, x)| \leq \gamma|x|$ whenever $|x| \leq \sigma_{2}$, uniformly in $t$. 
Choose $\varepsilon_{0}=\min \left(\sigma_{1}, \sigma_{2}\right)$ and for $0 \leq \varepsilon \leq \varepsilon_{0}$ choose $\delta \leq(1-K \zeta) \frac{\varepsilon}{2}$.

Then for $\varphi \in S_{\varepsilon}$ and $\|f\| \leq \delta$ one has from (4.1)

$$
|(T \varphi)(t)| \leq\|f\|+\int_{0}^{t}|v(t, s)| \gamma|\varphi(s)| d s+\int_{0}^{\infty}|k(t, s)| \zeta|\varphi(s)| d s,
$$

so that, using (2.4) and (2.8),

$$
\|(T \varphi)(t)\| \leq \delta+\frac{1-K \zeta}{2 B} \cdot \varepsilon B+\zeta \varepsilon K \leq \varepsilon
$$

and thus $7 S_{\varepsilon} \subseteq S_{\varepsilon}$.

(ii) $T$ is continuous on $S_{\mathrm{\varepsilon}}$.

This fact is proved exactly as in the proof of (ii) Theorem 1.

(iii) The function in the image set $T S_{\varepsilon}, 0 \leq \varepsilon \leq \varepsilon_{0}$, are bounded and equicontinuous at every point of $[0, \infty)$.

The boundedness is already established in (i). Let $t_{0} \in[0, \infty)$ and let $t>t_{0}$

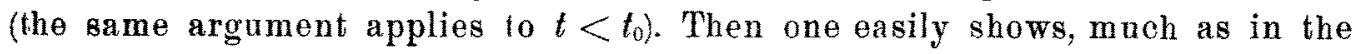
proof of (iii) Theorem 1:

$$
\begin{aligned}
\left|(T \varphi)(t)-(T \varphi)\left(t_{0}\right)\right| & \leq\left|f(t)-f\left(t_{0}\right)\right|+\gamma \varepsilon_{0}\left\{\int_{0}^{t}\left|v(t, s)-v\left(t_{0}, s\right)\right| d s\right. \\
& \left.+\int_{0}^{t}|v(t, s)| d s\right\}+\zeta \varepsilon_{0} \int_{0}^{\infty}\left|k(t, s)-k\left(t_{0}, s\right)\right| d s .
\end{aligned}
$$

Let $\eta>0$ be given. Choose $\delta_{1}>0$ so small that

$$
\left|f(t)-f\left(t_{0}\right)\right| \leq \eta / 3 \text { whenever }\left|t-t_{0}\right| \leq \delta_{1}
$$

choose $\delta_{2}>0($ by (2.5)) so small that

$$
\left|\int_{0}^{t_{0}}\right| v(t, s)-v\left(t_{0}, s\right) \| d s+\int_{t_{0}}^{t}\left|v(t, s)^{\prime} d s\right| \leq \frac{\eta}{3\left(1+\gamma \varepsilon_{0}\right)}
$$

whenever $\left|t-t_{0}\right| \leq \delta_{2}$; finally choose $\delta_{3}>0$ so small that (by (2.9))

$$
\int_{0}^{\infty}\left|k(t, s)-k\left(t_{0}, s\right)\right| d s \leq \frac{\eta}{3\left(1+\zeta \varepsilon_{0}\right)} \text { whenever } \| t-t_{0} \mid \leq \delta_{3} .
$$


Then for $\left|t-t_{0}\right|<\delta$ one has $\left|(T \varphi)(t)-(T \varphi)\left(t_{0}\right)\right| \leq \eta$, where $\delta=\min \left(\delta_{1}, \delta_{2}, \delta_{3}\right)$. Thus by the SOHĀDER-TXOHONOFF fixed point theorem there exists a function $x(t) \in S_{\varepsilon}$ such that $T x=x$ (i.e. $x(t)$ satisfies (2.7)) and $\|x\| \leq \varepsilon$. This establishes the first statement in Theorem 2. The proof that $\lim _{t \rightarrow \infty} x(t)=0$, under the additional hypothesis stated, may be carried out in two ways, much the same as that of Corollary 1.1 .

5. Proof of Theorem 3. - Let $y^{\prime}(t)$ be a bounded solution of (1.2) with $\|y\| \leq K$, for some $K>0$. We first establish the existence of a bounded solution $x(t)$ of (1.1). Let $R$ be the operator defined by (3.2) acting on the space $B O$ and let

We assert:

$$
S_{K}=\{\varphi: \varphi(t) \in B C, \quad\|\varphi\| \leq 2 K\} .
$$

(i) $R S_{K} \subset S_{K}$.

For, if $\|\varphi\| \leq 2 K$, then from $(3.2),(2.4)$ and $H_{4}$ one has

$$
\begin{aligned}
|(R \varphi)(t)| \leq & |y(t)|+\int_{0}^{t}|r(t, s)||g(s, \varphi(s))| d s \\
& \leq\|y\|+\int_{0}^{t}|r(t, s)| \lambda(s)|\varphi(s)| d s \leq K+2 K\|\lambda\| \leq 2 K .
\end{aligned}
$$

Thus $\|R \varphi\| \leq 2 H$. (This is the only place where assumption $\|\lambda\| B \leq \frac{1}{2}$ is used).

(ii) $R$ is continuous on $S_{K}$.

This is proved exactly as (ii) in the proof of Theorem 1.

(iii) The functions in the image set $R S_{K}$ are bounded and equicontinuous at every point of $[0, \infty)$. The boundedness has already been proved in (i). For the equicontinuity one has from $(3.2)$ and $H_{4}$ for $t>t_{0}$ (same argument applies to $t<t_{0}$ ):

$$
\begin{aligned}
& \left|(R \varphi)(t)-(R \varphi)\left(t_{0}\right)\right| \leq\left|y(t)-y\left(t_{0}\right)\right|+ \\
& \left.\sup _{0 \leq s \leq t}|g(s, \varphi(s))|\left|\int_{0}^{t_{0}}\right| r\left(t_{0}, s\right)-r(t, s)\left|d s+\int_{t_{0}}^{t}\right| r(t, s) \mid d s\right] \\
& \left.\leq\left|y(t)-y\left(t_{0}\right)\right|+2 \Lambda K\left|\int_{0}^{t_{0}}\right| r\left(t_{0}, s\right)-r(t, s)\left|d s+\int_{t_{0}}^{t}\right| r(t, s) \mid d s\right],
\end{aligned}
$$


where $\Lambda=\|\lambda\|$. Given $\varepsilon>0$ choose $\delta_{1}>0$ such that

$$
\left|y(t)-y\left(t_{0}\right)\right| \leq \frac{\varepsilon}{2} \text { for }\left|t-t_{0}\right| \leq \delta_{1}
$$

and choose $\delta_{2}>0$ such that (see $(2.5)$ )

$$
\int_{0}^{t_{0}}\left|r(t, s)-r\left(t_{0}, s\right)\right| d s+\int_{t_{0}}^{t}|r(t, s)| d s \leq \frac{\varepsilon}{4 K \Lambda} \text { for }\left|t-t_{0}\right| \leq \delta_{2} .
$$

Then for $\left|t-t_{0}\right| \leq \delta=\min \left(\delta_{1}, \delta_{2}\right)$ one has $\mid(R \varphi)(t)-(R \varphi)\left(t_{0}\right) \| \leq \varepsilon$ which proves the equicontinuity.

Thus by the Schadder-Tychonofe theorem the mapping $R$ has a fixed point in $S_{K}$, i.e. there exists a function $\tilde{\varphi} \in S_{K}$ such that

$$
\tilde{\varphi}(t)=y(t)-\int_{0}^{t} r(t, s) g(s, \tilde{\varphi}(s)) d s \quad(0 \leq t<\infty)
$$

and because of the equivalence of (2.3) and (1.1), $\varphi$ is a bounded solution of (1.1).

We next prove that $\lim _{t \rightarrow \infty}(\varphi(t)-y(t))=0$. Let $\varepsilon>0$ be given. Since $\lambda(t) \rightarrow 0$ as $t \rightarrow \infty$ choose $T>0$ such that

$$
|\lambda(t)| \leq \frac{\varepsilon}{4 K B} \quad(t \geq T)
$$

where $B$ is the constant in (2.4). Choose $T_{1} \geq T$ such that (see (2.6))

$$
\int_{0}^{T}|\boldsymbol{r}(t, s)| d s \leq \frac{\varepsilon}{4 \Lambda K} \quad\left(t \geq T_{1}\right)
$$

From $(2.3),\left(H_{4}\right)$ and $\|\tilde{\varphi}\| \leq 2 K$ one has for $t \geq T$

$$
\begin{aligned}
& |\tilde{\varphi}(t)-y(t)| \leq \int_{0}^{T}|r(t, s)| \lambda(s)|\tilde{\varphi}(s)| d s+\int_{T}^{t}|r(t, s)| \lambda(s)|\tilde{\varphi}(s)| d s \\
& \quad \leq 2 K \Lambda \int_{0}^{T}|r(t, s)| d s+2 K \sup _{T \leq t \leq \infty} \lambda(t) \int_{0}^{t}|r(t, s)| d s .
\end{aligned}
$$


Using (5.2) to estimate the first term and $(5.1)(2.4)$ the second term one obtains

$$
\tilde{\varphi}(t)-y(t)<\frac{\varepsilon}{2}+\frac{\varepsilon}{2}=\varepsilon \quad\left(t \geq T_{1}\right)
$$

Since $\varepsilon>0$ is arbitrary this proves the assertion and completes the proof of the first part.

To prove the converse let $u(t)$ be a bounded solution of (1.1) (i.e. of (2.3)). Define

$$
v(t)=u(t)+\int_{0}^{t} r(t, s) g(s, u(s)) d s
$$

Evidently by $\left(H_{4}\right)$ and (2.4)

$$
|v(t)| \leq\|u\|+\Lambda B\|u\|<\infty \quad(0 \leq t<\infty)
$$

so that $v$ is bounded. Wo assert

(i) $\lim _{t \rightarrow \infty}(v(t)-u(t))=0$

Let $\varepsilon>0$ be given. Let $\|u\|=K$. Define $T, T_{1}$ as in (5.1), (5.2). Then from (5.3), $H_{4}$ one has for $t \geq T$

$$
|v(t)-u(t)| \leq \int_{0}^{T}|r(t, s)| \lambda(s)|u(s)| d s+\int_{T}^{t}|r(t, s)| \lambda(s)|u(s)| d s,
$$

and from (2.4), (2.6), (5.1), (5.2) for $t>T_{1}$

$$
|v(l)-u(t)| \leq \Lambda K \frac{\varepsilon}{4 K \Lambda}+\frac{\varepsilon}{4 K B} K B=\frac{\varepsilon}{2}
$$

Since $\varepsilon>0$ is arbitrary this proves the assertion.

(ii) $v(t)$ is a solution of (1.2).

For, using successively (5.3), FubinI's theorem, (2.1), (1.1), and again (5.3) one has:

$$
\begin{aligned}
& f(t)-\int_{0} a(t, s) v(s) d s \\
= & \left.f(t)-\int_{0}^{t} a(t, s) u^{\prime} s\right) d s-\int_{0}^{t} a(t, s)\left\{\int_{0}^{s} r(s, \sigma) g\left(\sigma, u_{0}^{\prime}(\sigma)\right) d \sigma\right\} d s
\end{aligned}
$$




$$
\begin{aligned}
& =f(t)-\int_{0}^{t} a(t, s) u(s) d s-\int_{0}^{t}\left\{\int_{\sigma}^{t} a(t, s) r(s, \sigma) d s\right\} g(\sigma, u(\sigma)) d \sigma \\
& =f(t)-\int_{0}^{t} a(t, s) u(s) d s-\int_{0}^{t} a(t, \sigma) g(\sigma, u(\sigma)) d \sigma+\int_{0}^{t} r(t, \sigma) g(\sigma, u(\sigma)) d \sigma \\
& =u(t)+\int_{0}^{t} r(t, \sigma) g(\sigma, u(\sigma)) d \sigma=v(t)
\end{aligned}
$$

which shows that $v$ satisfies (1.2).

This completes the proof of Theorem 3 .

6. Proof of Theorem 4. - Let $y(t) \in B C$ be a solution of (1.2). Let $x(t)$ be a local, continuous solution of (1.1) (equivalently of (2.3)) existing on some interval $0 \leq t \leq \tau$. The existence of such a solution can be established in several (classical) ways; assumption (2.5) insures the continuity of the solution. Let

$$
\|x\|_{\tau}=\sup _{0 \leq t \leq \tau}|x(t)|
$$

We show that $\|x\|_{\tau}$ satisfies an a priori bound independent of $\tau$.

From (2.3), (2.4) and (2.13) one has

$$
\begin{aligned}
\|x\|_{\tau} & \leq\|y\|+\int_{0}^{\tau}|r(\tau, s)| \lambda(s)|x(s)|^{\sigma} d s \\
& \leq\|y\|+\|x\|_{\tau}^{\sigma} \Lambda B,
\end{aligned}
$$

where $\Lambda=\|\lambda\|$. Using Young's inequality for positive real numbers $a$ and $b$ :

$$
a b \leq \frac{a^{p}}{p}+\frac{b^{q}}{q} \quad\left(\frac{1}{p}+\frac{1}{q}=1\right)
$$

with $p=\frac{1}{\sigma}, q=\frac{1}{1-\sigma}, 0<\sigma<1$, one obtains

Thus

$$
\|x\|_{\tau} \leq\|y\|+\sigma \mid x \|_{\tau}+(1-\sigma)(B \Lambda)^{\frac{1}{1-\sigma}}
$$

$$
\|x\|_{\tau} \leq(1-\sigma)^{-1}\|y\|+(B \Lambda)^{\frac{1}{1-\sigma}}
$$


If $\sigma=0$, the estimate (6.1) also holds, as may be seen directly from (2.3). Clearly, the right hand side of $(6.1)$ is independent of $\tau$.

A standard continuation argument for Volterra equations (see eg [10; lemma 1.2]) shows that the local solution can be continued to the right to the interval $0 \leq t \leq \infty$, and that the continuation, which we again call $x(t)$ for simplicity, satisfies the a priori bound (6.1). In particular $x(t) \in B C$ and $\|x\| \leq K$, where $K$ is the right hand side of $(6.1)$.

To oomplete the first part of the proof we assert that $x(t)-y(t) \rightarrow 0$ as $t \rightarrow \infty$. Let $\varepsilon>0$ be given. Since $\lambda(t) \rightarrow 0$ choose $T>0$ such that

$$
\lambda(t) \leq \frac{\varepsilon}{2 K^{\sigma} B} \quad(t \leq T)
$$

where $B$ is defined by (2.4). Choose $T_{1} \geq T$ such that (by (2.6))

$$
\int_{0}^{T}|r(t, s)| d s \leq \frac{\varepsilon}{2 \Lambda K^{\sigma}} \quad\left(t \geq T_{1}\right)
$$

From (2.3) and (2.12) one has for $t \geq T$

$$
|x(t)-y(t)| \leq \int_{0}^{T}|r(t, s)| \lambda(s)|x(s)|^{\sigma} d s+\int_{T}^{t}|r(t, s)| \lambda(s)|x(s)|^{\sigma} d s .
$$

Since $\|x\| \leq K$ this yields

$$
|x(t)-y(t)| \leq \Lambda K^{\sigma} \int_{0}^{T}|r(t, s)| d s+K^{\sigma} \sup _{T \leq t<\infty} \lambda(t) \int_{0}^{t}|r(t, s)| d s .
$$

Using (6.3) to estimate the first term and (6.2), (2.4) to estimate the second term one obtains

$$
|x(t)-y(t)| \leq \Lambda K^{\sigma} \frac{\varepsilon}{2 \Lambda K^{\sigma}}+K^{\sigma} \frac{\varepsilon}{2 K^{\sigma} B} B=\varepsilon \quad\left(t \geq T_{1}\right) .
$$

Since $\varepsilon>0$ is arbitrary, this proves the assertion and completes the first part of the proof.

To prove the converse, namely that the existence of a bounded solution $u(t)$ of (1.1) implies the existence of a bounded solution $v(t)$ of (1.2) such that $u(t)-v(t) \rightarrow 0$ as $t \rightarrow \infty$, proceeds exactly as in the second part of the proof of Theorem 3, Section 5, using (6.2), (6.3) in place of $(5.1),(5.2)$ respectively. This completes the proof of Theorem 4 . 


\section{REFERENCES}

[1] R.K. Miller, J.A. Norer, J.S. W. Wong, Perturbations of Volterra Integral equa. tions, J. Math. Anal. Appl. 25 (1969), pp. 676-691.

[2] - - A stability theorem for nonlinear mixed integral equations, J. Math. Anal. Appl. 200 (1969), pp. 446.449.

[3] A. Strauss, On a perturbed Volterra integral equation, J. Math .Anal. Applic. 30 (1970), pp. 564.575 .

[4] N. Levinson, The asymptotic behaviour of a system of linear differential equations, Am. J. Math. 68 (1946), pp. 1-6.

[5] H. WEYL, Comment on the preceding paper, An. J. Math. 68 (1946), pp. 7.12.

[6] F. Brauer and J.S. W. WovG, On the asymptotic relationships between solutions of of two systems of ordinary differential equations, J. Diff. Eq. 6 (1939), pp. 527.543.

[7] W. A. CoPper, Stability and Asymptotic Behaviour of Differential Equations, Heath Math. Monograph, D. C. Heath \& Co., Boston, 1960.

[8] R. K. MIller, Admissibility and nonlinear Volterra equations, Proc. AMS 25 (1970), pp. 65.71.

[9] J.J. Levis, Boundedness and oscillation of some Volterra and delay equations, J. Diff. Eq. 5 (1969), pp. 369-398.

[10] J.A. NoHEL, Some problems in nonlinear Volterra equations, Bull. AMS (1969), pp. 323.329.

Un. of Wiscounsin, Madison (U.S.A.). 